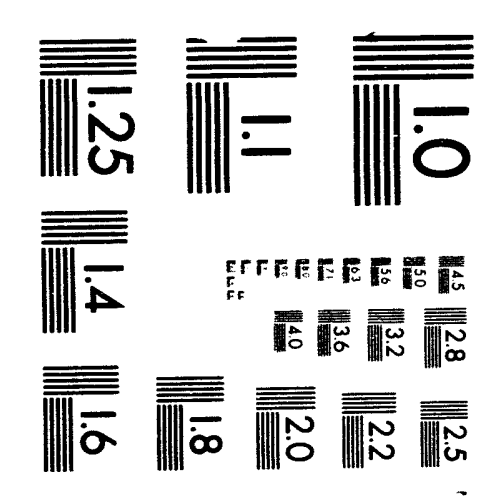



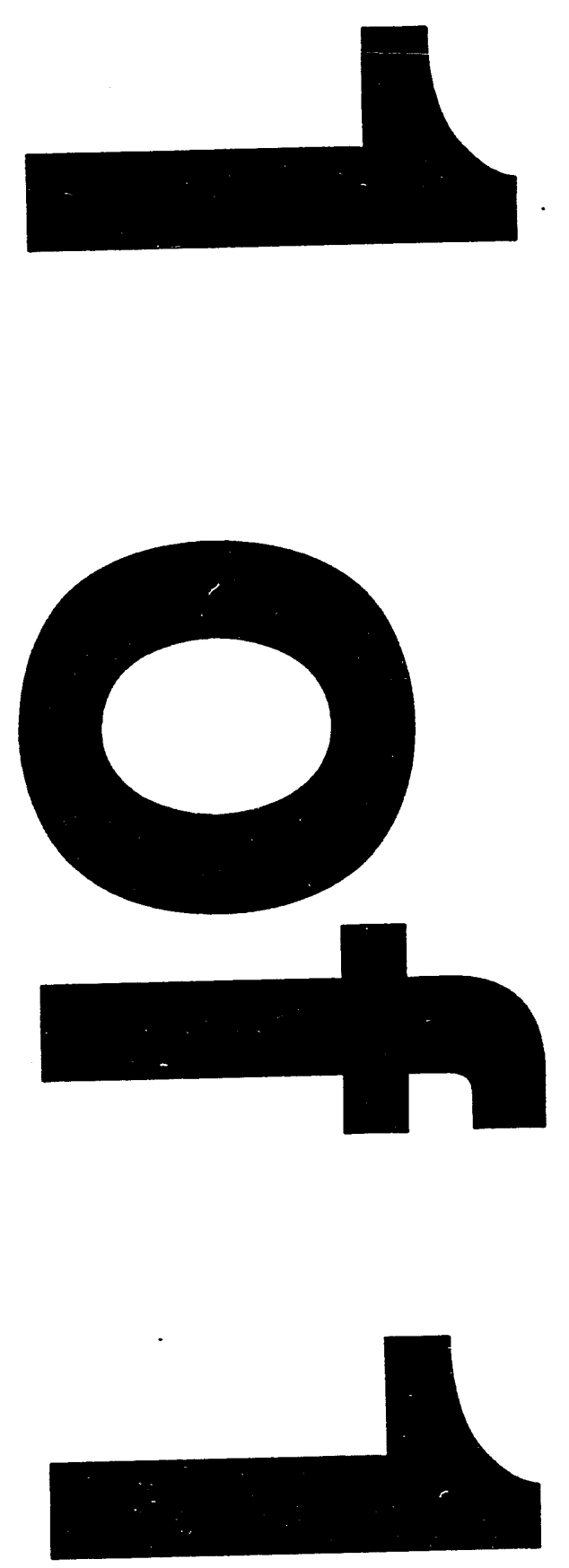


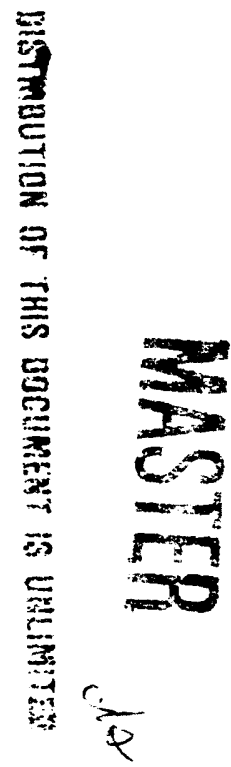

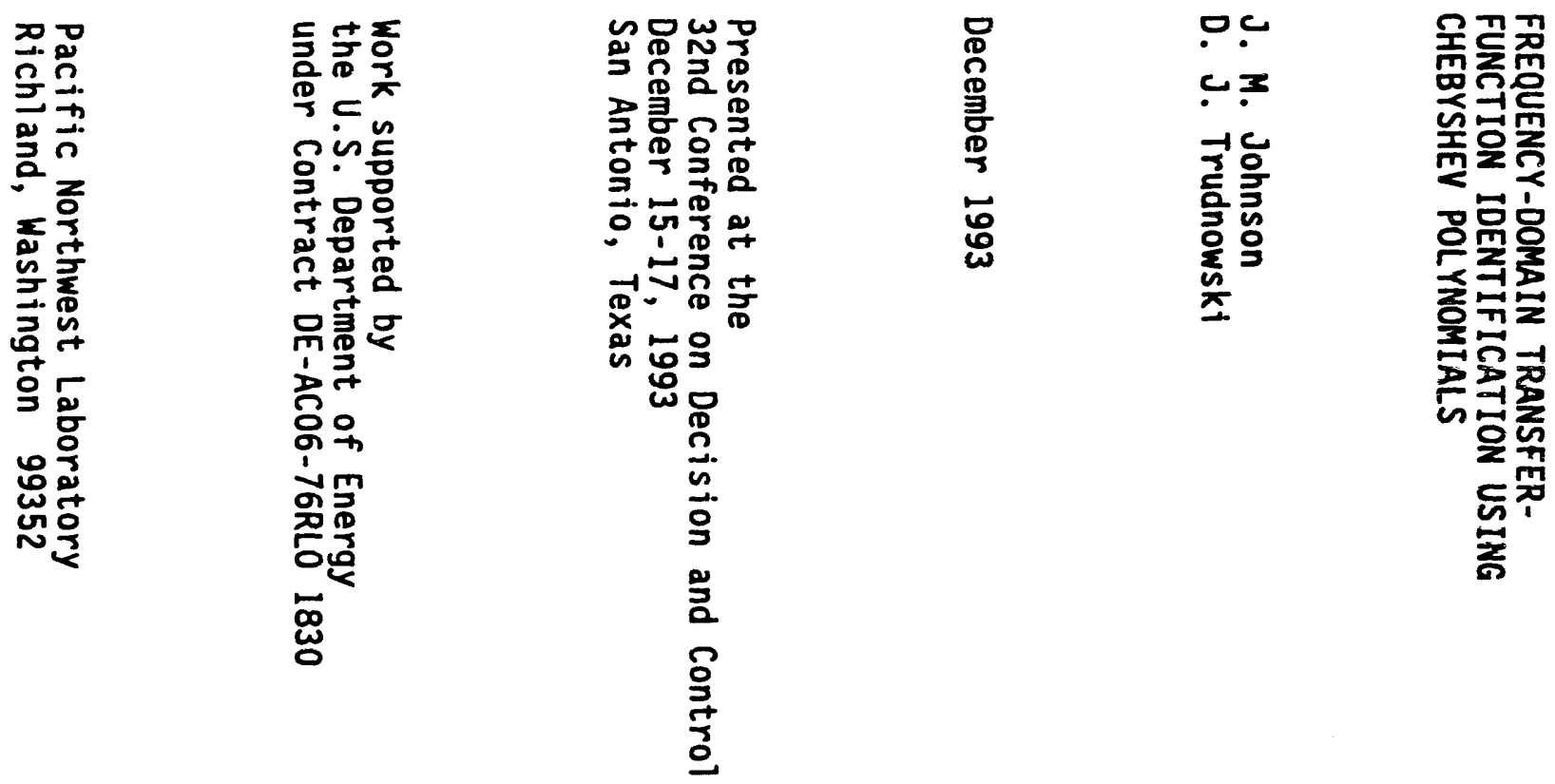

\section{DISCLAIMER}

This report was prepared as an account of work sponsored by an agency of the United States Government. Neither the United States Government nor any agency thereof, nor any of their employees, makes any warranty, express or implied, or assumes any legal liability or responsibility bility for the accuracy, completer, it use would not infringe privately owned rights. Referprocess disclosed, or represents that its use would not infringe prively ow ence herein to any specific commercial produch, process, or service by trade name. trademark, manufacturer, or otherwise does not necessarily constitute or imply its endorsement, recommendation, or favoring by the United States Government or any agency thereor. The views and opinions authors expressed herein do not necessarily state or reflect those of the United States Government or any agency thereof.

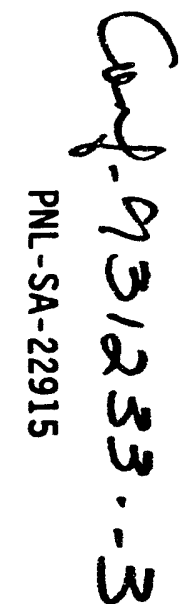




\title{
FREQUENCY-DOMAIN TRANSFER-FUNCTION IDENTIFICATION USING CHEBYSHEV POLYNOMIALS
}

\author{
J. M. Johnson and D. J. Trudnowski \\ Pacific Northwest Laboratory ${ }^{1}$ \\ Richland, Washington 99352
}

\begin{abstract}
An approach is proposed for identifying a linear single-input single-output model from the frequency response of a system with unknown order. The approach involves representing the transfer function as the ratio of linearly-combined Chebyshev polynomials and solving an over-determined linear equation set. The method is compared with two other previously proposed techniques and shown to perform very well under noisy data conditions.
\end{abstract}

\section{Introduction}

A fundamental problem often encountered in studying dynamic systems involves identifying a lir ar transfer-function model from a system's frequency response. Consider a general singleinput single-output system with the Laplace-domain transfer function $G(s)$. The objective is to fit the linear model

$$
G_{M}(s)=\frac{b_{0} \phi_{0}(s)+b_{1} \phi_{1}(s)+\cdots+b_{m} \phi_{m}(s)}{a_{0} \theta_{0}(s)+a_{1} \theta_{1}(s)+\cdots+a_{n} \theta_{n}(s)}
$$

where $a_{i}, b_{i} \in \Re$ and $\phi_{i}=\theta_{i}=s^{i}$ to the system's frequency response $G(j \omega)$ such that $\left\|G(j \omega)-G_{M}(j \omega)\right\|$ is minimized. Unfortunately, this is not a linear problem. Therefore, we compromise and minimize $\|A(j \omega) G(j \omega)-B(j \omega)\|$. This second cost function is termed the equation error problem [1].

Assume that $N$ samples of $G(j \omega)$ are known; i.e, $G\left(j \omega_{k}\right)$, $\mathrm{k}=0,1, \cdots, N-1$. Also, assume that $N \geq n+m+2$. In the practical case, $m$ and $n$ are rarely known. How one can estimate these is discussed in Section III

\section{Fitting Algorithms}

A number of methods are possible for solving the above problem. In this section, we describe three approaches.

\section{Method 1: Linear Least-Squares}

A straightforward method of identifying an optimal model is to set $\phi_{i}=\theta_{i}=s^{j}$ and directly fit equation (1) to the system samples. Let $G_{k}$ and $G_{M k}$ represent $G\left(j \omega_{k}\right)$ and $G_{M}\left(j \omega_{k}\right)$. respectively. Equation (1) is expanded and $G_{k}$ substituted for $G_{M k}$, resulting in an over-determined set of equations

'Operated for the U. S. Department of Energy by Battelle Memorial Institute under Contract DE-AC06-76RLO 1830. Portions of this research were supported by the Northwest College and University Association for Science (Washington State University) and Grant DE-FG06-89ER-75522 with the U.S. Department of Energy.

$$
\left[\begin{array}{ll}
J & K
\end{array}\right]\left[\begin{array}{l}
\underline{a} \\
\underline{b}
\end{array}\right]=\left[\begin{array}{l}
0 \\
0
\end{array}\right]
$$

where $J$ is an $N \times(n+1)$ matrix whose elements are given by $J_{i k}=\left(j \omega_{i-1}\right)^{k-1} \cdot G_{i-1}, K$ is an $N \times(m+1)$ matrix whose elements are given by $K_{i k}=-\left(j \omega_{i-1}\right)^{k-1} G_{i-1}, \underline{a}=\left[\begin{array}{llll}a_{0} & a_{t} & \cdots & a_{n}\end{array}\right]^{\mathrm{T}}$ and similarly for $\underline{b}$. A least-squares solution is obtained by simultaneously solving the real and imaginary parts of (2) using the Moore-Penrose pseudoinverse [2]. In this paper, we use a singular value decomposition approach to compute the pseudoinverse.

Method 2: Least-squares using Chebyshev Polynomials Recognizing that the system described by (1) is simply the ratio of two linear combinations of polynomials, Richardson and Formenti [3] proposed letting $\phi_{i}$ and $\theta_{i}$ be general polynomials of order $i$. Furthermore, $\left\{\phi_{i}, \mathrm{i}=0,1, \cdots, m\right\}$ and $\left\{\theta_{i}, \mathrm{i}=0,1\right.$, $\cdots, n\}$ are orthogonal sets. This formulation is limiting because $\phi_{i}$ and $\theta_{i}$ arc evaluated at complex values for $s=j \omega$, while many well-known polynomial sets are orthogonal for real arguments.

To alleviate this problem, we define a normalized frequency by $\hat{\omega}=s / j \Omega$ where $\left.\Omega \Delta \max || \omega_{k} \mid ; k=0,1, \cdots, N-1\right\}$. We then set $\phi_{i}=\theta_{i}=J^{3} \cdot T_{i}(\hat{\omega})$ in $(1)$, where $T_{i}(\hat{\omega})=\cos \left(i \cos ^{-1}(\hat{\omega})\right)$ is the $i$ th Chebyshey polynomial. Equation (1) is expanded and solved as in Method 1 except this time $J_{i k}=j^{k-1} \cdot T_{k-1}\left(\hat{\omega}_{i-1}\right) \cdot G_{i-1}$ and $K_{i k}=-f^{t-1} \cdot T_{k-1}\left(\hat{\omega}_{i-1}\right) . J$ and $K$ are again $N \times(n+1)$ and $N \times(m+1)$, respectively. Vectors $a$ and $b$ obtained here contain the coefficients for $\phi_{i}=\theta_{i}=j^{i} T_{i}(\omega)$. The coefficients for $\phi_{i}=\theta_{i}=s^{s}$ are calculated by equating (1) written with $\phi_{i}=\theta_{i}=j^{i} T_{i}(\hat{\omega})$ to (1) written with $\phi_{i}=\theta_{i}=s^{i}$ for $s=j \omega$, which results in a linear transformation matrix involving the Chebyshev polynomial coefficients.

\section{Method 3: Adcock's Method}

A third fitting technique, used here for comparison, is the method in [4]. The model transfer function is represented as in Method 2; but, here the cost function is $\|W(j \omega)(A(j \omega) G(j \omega)-B(j \omega))\|$, where $W(j \omega)$ is a weighting function. Using the derivation path in [4], minimizing this cost function results in an $(n+m+2) \times(n+m+2)$ set of linear equations for the Chebyshev series coefficients in equation (1).

\section{Discussion}

Several issues must be addressed when using and comparing the fitting algorithms. A question is: which formulation provides better numerical results? This issue is discussed here; also discussed is the problem of choosing $n$ and $m$. 
As recognized by Richardson and Formenti [3], the Method 1 algorithm has poor numerical properties. This is primarily caused by the basis functions $s^{0}, s^{1}, \cdots, s^{k}$ not forming an orthogonal set [5] thus causing the matrix in (2) to be numerically ill-conditioned. Methods 2 and 3 address the numerical sensitivity problem by normalizing frequencies and using the orthogonal Chebyshev polynomials as the basis functions. The advantage of this is discussed in [4].

The solutions for Methods 1 and 2 are least squares solutions obtained by calculating the Moore-Penrose pseudoinverse of the matrix $[J K]$ in (2), which has dimensions $N \times(n+m+2)$. The solution for Method 3 requires inversion of an $(n+m+2) \times(n+m+2)$ matrix, which is generally of substantially smaller dimension.

The difference between Methods 2 and 3 is in how the leastsquares problem is solved. With Method 2, an over-determined approach is taken. In the presence of additive Gaussian white noise, this method will produce optimal parameter estimates. Method 3 uses a different approach that requires squaring of the data $G_{k}$. Because of this squaring, noise associated with the data causes a bias resulting in non-optimum parameter estimates. To alleviate this problem, Adcock [4] recommends subtracting the noise variance from the squared data but in many cases, the variance is unknown.

With all the proposed algorithms, $n$ and $m$ are assumed known. In many identification problems, the order is unknown. It is our experience that it is best to over-estimate the model order and use a model-order reduction technique such as that described in [6] to remove extra terms. Under noisy conditions, the extra degrees of freedom provided by overestimating the model order tend to fit to the effects of the noise and nonlinearities, allowing the remaining parameters to fit to the actual signal. This same effect has been observed in fitting to time-domain data [7]. The following example demonstrates these and other effects.

\section{Example:}

Consider the 9 pole, 8 zero system described in Table I, which is a reduced-order linearized version of a power system electromechanical oscillatory model.

Table I Poles, zeros and gain of example function.

\begin{tabular}{|c|c|c|}
\hline Poles & Zeros & DC Gain \\
\hline-10.053 & $-0.8 \pm j 3.456$ & 44.092 \\
$-0.5 \pm j 2.199$ & $-0.15 \pm j 4.084$ & \\
$-0.25 \pm j 2.827$ & $-0.30 \pm j 5.655$ & \\
$-0.10 \pm j 5.027$ & $-0.60 \pm j 9.425$ & \\
$-0.05 \pm j 6.283$ & & \\
\hline
\end{tabular}

The data set used is 2048 impulse response samples calculated at intervals of 0.05 seconds. Gaussian white noise is added to these so that the signal-to-noise ratio is $20 \mathrm{~dB}$. The discrete fourier transform (DFT) is then used to estimate the frequency response. Both the noise and the DFT tend to cause errors in this response. The three fitting routines are used with $N=256$ samples at a sample frequency of $2.5 \cdot(2 \pi) / 256 \mathrm{rad} / \mathrm{sec}, n=30$, and $m=29$. The fits are shown in Figure 1. The Method 2 algorithm accurately identifies all the terms except the zeros at $s=-0.60 \pm j 9.425$. The Method 3 algorithm is less effective; the Method 1 algorithm does not identify any terms.
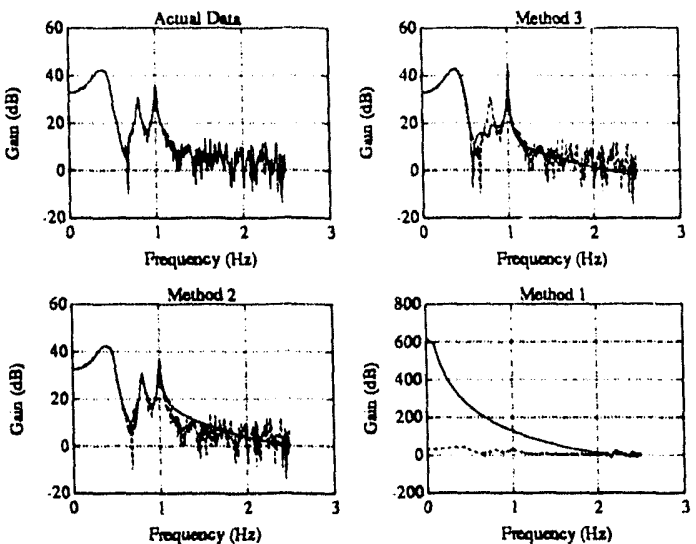

Figure 1 Magnitude plots of identified transfer function fits.

\section{Conclusion}

This paper has demonstrated the advantage of formulating a transfer function model as a ratio of linear-combined Chebyshev polynomials when fitting to a system's frequency response. From the example, it appears that solving the system using a linear least-squares approach results in a more accurate model than solving the system as proposed in [4]. The penalty is that a more numerically intensive problem must be solved; but with a modern computer this is often not an issue. The value of overestimating the model order (even when it is known) during fitting has also been demonstrated.

\section{References}

[1] T. Soderstrom and P. Stocia, System Identificatiom Prentice Hall, New York, 1989.

(2) P. Lancaster and M. Tismenetsky, The Theory of Matrices, Harcourt Brace Jovanovich, New York, 1985.

(3) M. H. Richardson and D. L. Formenti, "Parameter Estimation from Froquency Response Measurements Using Rational Fraction Polynomials," In Proceedings of International Modal Analysis Conference, pp. 167-181, Schenectady, New York, 1982.

[4] J. L. Adcock, "Curve Fitter for Pole-Zero Analysis," Hewlett-Packard Jowrnal. Vol. 38, no. 12. Jan 1987.

(5) L. W. Johnson and R. D. Press, Numerical Analysis, Addison-Wesley, Reading, Mass, 1982.

[6] D. J. Trudnowski, "Order Reduction of Large-Scale Linear Oscillatory System Models," Paper No. 93WM209-7PWRS, IEEE Power Engineering Society Winter Power Meeting, Columbus, Ohio, Feb. 1993. To appear in IEEE Trans. on Power Systems.

[7] D. W. Tufts and R. Kumaresan, "Estimation of Frequencies of Multiple Sinusoids: Making Linear Prediction Perform Like Maximum Likelihood," Proceedings of the IEEE, Vol. 70, No. 9, pp. 975-989, 1982. 

